\title{
Alterações radiográficas do aparato podotroclear de equinos da Polícia Militar de Minas Gerais sem sinais clínicos de doença do osso navicular
}

\author{
[Radiographic changes in the podotroclear apparatus from Minas Gerais State Military \\ horses without clinical signs of navicular disease] \\ D.S.D. Azevedo ${ }^{1}$, V.F. Xavier $^{2}$, R.M. Ribeiro ${ }^{2}$, S.B. Villela ${ }^{1}$, T.C. Castro $^{1}$, R.A.M. Vieira ${ }^{1}$, \\ I.R. Lima ${ }^{2}$, C.A. Marval ${ }^{3}$, R.R. Faleiros ${ }^{4 *}$ \\ ${ }^{1}$ Aluno de graduação - Escola de Veterinária - UFMG - Belo Horizonte, MG \\ ${ }^{2}$ Aluno de pós-graduação - Escola de Veterinária - UFMG - Belo Horizonte, MG \\ ${ }^{3}$ PUCMINAS e PMMG - Belo Horizonte, MG \\ ${ }^{4}$ Escola de Veterinária - UFMG - Belo Horizonte, MG
}

\section{RESUMO}

Os objetivos deste experimento foram identificar e associar alterações radiográficas do aparato podotoclear de equinos do Regimento de Cavalaria Alferes Tiradentes da Policia Militar do estado de Minas Gerais sem histórico e sinais clínicos de doença do osso navicular. Foi avaliado um total de 33 equinos, de ambos os sexos, com idade entre 10 e 20 anos. Os dígitos torácicos foram radiografados de forma padronizada nas projeções lateromedial (LM), dorsoproximal palmarodistal $65^{\circ}$ (DPPD) e palmaroproximal palmarodistal (SK). A radiopacidade medular aumentada em projeção SK foi a principal alteração radiográfica detectada. Essa alteração foi associada a maior número de invaginações sinovais, a maior espessura de cortical em relação à medular em exposição SK e a maior relação corticomedular em exposição LM $(\mathrm{P}<0,05)$. Esses achados indicam uma predisposição da população equina para desenvolver a síndrome do osso navicular, possivelmente associada ao trauma repetitivo promovido pelo constante trabalho em piso duro.

Palavras-chave: síndrome do osso navicular, claudicação, radiologia

\begin{abstract}
The aims of this study were to identify and associate radiographic changes of podotoclear apparatus in horses from the Tiradentes Calvary Regiment of the Military Police of Minas Gerais State without history and clinical signs of navicular disease. 33 horses from both sexes, aged between 10 and 20 years were evaluated. The thoracic digits were radiographed in a standardized manner in lateralmedial (LM), palmaroproximaldistodorsal $65^{\circ}(D P P D)$ and palmaroproximal-distopalmar (SK) projections. The increased medullary radiopacity in $S K$ projection was the main radiological change detected and was associated with a higher number of synoval invaginations, increased cortical thickness in relation to medulla in SK exposure and increased corticomedullar in LM exposure $(P<0.05)$. These findings indicate a predisposition of this population to develop navicular syndrome, which is possibly associated with repetitive trauma promoted by constant work on hard floors.
\end{abstract}

Keywords: navicular syndrome, lameness, radiology

\section{INTRODUÇÃO}

A utilização do cavalo pela Polícia Militar no Brasil remonta a meados do século XIX, com seu uso como meio de locomoção para patrulhamento, realização de diligências, escoltas e apoio operacional às tropas a pé, com liberdade para percorrer terrenos acidentados, inóspitos e de difícil acesso, disponibilizando velocidade e rapidez de ação. Os equinos podem ainda acrescentar um fator social à Policia Militar, uma vez que os cavalos possuem uma relação com a população pela atração natural das crianças em relação ao animal, bem como uma importância histórica com o desenvolvimento humano (Rivelles, 2011).

Recebido em 12 de abril de 2014

Aceito em 4 de maio de 2015

*Autor para correspondência (corresponding author)

E-mail: faleirosufmg@gmail.com 
Entre as lesões que afetam os equinos, as afecções musculoesqueléticas são a segunda maior causa de óbito nessa espécie, totalizando $15,8 \%$ dos diagnósticos das necropsias realizadas na Inglaterra durante o período entre 1958 e 1980 (Baker e Ellis, 1981). Em outro estudo retrospectivo realizado na Suécia durante o período correspondente ao intervalo dos anos de 1997 a 2000, foi observado que as doenças articulares foram as responsáveis pelas principais afecções observadas (Egenvall et al., 2006). A síndrome do osso navicular é responsável por um terço das claudicações em cavalos atletas (Turner, 1990; Pleasant e Crisman, 1997). Em uma pesquisa realizada em cavalos de rodeio, apartação, tambor e rédeas, a síndrome do osso navicular está entre as principais alterações do membro locomotor que acometem esses animais (DaBareiner et al., 2005).

Apesar de tamanho impacto negativo na criação de equinos, ainda pouco se sabe sobre a fisiopatologia e o diagnóstico precoce dessa afecção. Os achados radiográficos que têm sido compatíveis com a síndrome do osso navicular são o aumento de tamanho e alteração na forma dos forames distais, lise do córtex flexor, remodelagem das bordas distal ou proximal do osso navicular e perda da distinção corticomedular (Pleasant e Crisman, 1997). A alteração observada no limite córtico-medular está relacionada com processo de remodelação e reabsorção óssea, podendo ser classificada também como um sinal de lesão crônica do osso sesamoide distal (Hernández e Novales, 1999; Clercq et al., 2000; Pool, 1995).

Segundo informações do setor de Medicina Veterinária do Regimento de Cavalaria Alferes Tiradentes da Polícia Militar do estado de Minas Gerais, a principal causa de afastamento dos cavalos em serviço são as claudicações, sendo a doença do osso navicular uma ocorrência comum (DeMarval, 2013; comunicação pessoal). A fim de buscar um método de diagnóstico precoce da doença do osso navicular nessa população, o objetivo do presente estudo foi verificar indícios de alterações radiográficas precoces do aparato podotoclear e suas possíveis associações em equinos do Regimento de Cavalaria Alferes Tiradentes da Polícia Militar do estado de Minas Gerais sem histórico e sinais clínicos dessa afecção.

\section{MATERIAL E MÉTODOS}

Foi avaliado um total de 33 equinos, de ambos os sexos, com idade entre 10 e 20 anos, sem apresentar sinais clínicos de claudicação, estabulados no Regimento de Cavalaria Alferes Tiradentes da Polícia Militar do estado de Minas Gerais, de diferentes raças, sendo, na sua maioria, Crioulo e Brasileiro de Hipismo. Durante o período experimental, todos os animais foram submetidos ao mesmo manejo alimentar, em que eram oferecidos feno e concentrado três vezes ao dia, na quantidade de $6 \mathrm{~kg} / \mathrm{dia}$ e $4 \mathrm{~kg} / \mathrm{dia}$, respectivamente, e água e sal mineralizado ad libitum, de acordo com as recomendações estabelecidas no Equine Nutrient Requeriments, NRC (2007). O feno utilizado foi de gramínea Cynodon dactylon (L.) Pers.Var. "Coast cross". O regime de trabalho se caracterizava por escala de 12 horas de exercício de patrulhamento urbano, seguido por 36 horas de folga, durante todo o ano.

Para realização do estudo radiográfico, os dígitos torácicos foram desferrados e limpos. Por meio de um aparelho de RX portátil (ORANGE $1060 \mathrm{HF}$ Vet), foram obtidas projeções lateromedial (LM), dorsoproximal palmarodistal $65^{\circ}$ (DPPD) e palmaroproximal palmarodistal ("skyline" ou SK). Com ajuda da guia de luz ou laser pointer, manteve-se sempre a mesma distância do emissor ao dígito, e o fator de exposição utilizado foi de $68 \mathrm{KV}$ e $2 \mathrm{MAS}$ para todos os animais e exposições.

$\mathrm{Na}$ projeção LM, foram avaliados a presença ou ausência de entesófitos no ligamento ímpar (ELI), a relação corticomedular (RCM), a radiopacidade medular em uma escala de valores entre 0 e 5 ( 0 mais radioluscente e 5 mais radiopaco) e o contorno do osso navicular $(\mathrm{CN})$, classificando-o de 0 a 3 quanto a possíveis irregularidades: ausentes (0), discretas (1), moderadas (2) e intensas (3).

Na projeção DPPD, foram avaliados o número de invaginações sinoviais, a simetria óssea em uma escala de 0 a 3: simétrica (0) ou discreta (1), moderada (2) e intensa (3) assimetria, e a borda proximal do osso navicular, observando-se características quanto a sua forma: reta, côncava, convexa e ondulada.

$\mathrm{Na}$ projeção SKYLINE, foram avaliadas a radiopacidade da medular em relação à cortical, a 
relação entre as espessuras medular e cortical e a presença de irregularidades na cortical flexora. A radiopacidade da medular em relação à cortical foi classificada utilizando-se uma escala de valores entre 0 e 5 ( 0 para mais radioluscente e 5 para o mais radiopaco, ou seja, radiopacidade medular igual à cortical). A relação entre medular e cortical foi obtida pela mensuração das espessuras de cortical e medular, sempre no nível da crista flexora, obtendo-se, em seguida, o valor percentual da espessura de cortical em relação à da flexora (espessura da cortical x 100/espessura da medular). Já a cortical flexora foi classificada de 0 a 3 quanto à ausência (0) ou presença discreta (0), moderada (2) e intensa (3) de lesões.

Os dados foram tabulados, e as incidências dos achados e as médias dos parâmetros paramétricos foram calculadas para ambos os membros torácicos. Os lados direito e esquerdo foram comparados quanto à dispersão de frequência pelo teste de qui-quadrado e quanto à variância pelo teste $t$ de Student.

A fim de se verificarem possíveis efeitos entre as variáveis estudadas, foram formados grupos considerando diferentes características. $\mathrm{Na}$ exposição LM, os dígitos foram divididos em dois grupos quanto à presença ou não de irregularidades no contorno do osso navicular e em outros dois grupos quanto à presença ou não de entensiófitos no ligamento ímpar. $\mathrm{Na}$ exposição DPPD, os dígitos foram divididos em quatro grupos com relação à forma de sua borda proximal: reta, côncava, convexa e ondulada. Também nessa exposição, dividiram-se três grupos quanto ao grau de simetria óssea. Já na exposição Skyline, formaram-se três grupos quanto aos graus de radiopacidade da medular: reduzida (grau 1), normal (graus de 2 a 4) e aumentada (grau 5). Nessa mesma exposição, formaram-se dois grupos com base na presença ou não de irregularidades na cortical flexora. Considerando-se cada um dos aspectos, os grupos foram comparados entre si quanto aos valores das diversas variáveis estudadas, utilizando-se o teste $t$ de Student ou de análise de variância totalmente ao acaso para dados paramétricos e o teste de Mann-Whitney ou de Kruskal-Wallis para dados não paramétricos. Para todos os testes, considerou-se um nível de significância de $\mathrm{P}<0,05$.

\section{RESULTADOS}

As incidências totais dos diferentes graus, números e formas das variáveis estudadas estão representadas na Tab. 1. Não foram encontradas quaisquer diferenças estatísticas entre os lados direito e esquerdo.

Tabela 1. Incidência percentual de características radiográficas de ossos naviculares torácicos em equinos do Regimento de Cavalaria Alferes Tiradentes

\begin{tabular}{|c|c|c|c|c|c|c|}
\hline & $\begin{array}{c}0 \\
\text { (ausente) }\end{array}$ & $\begin{array}{c}1 \\
\text { (presente) }\end{array}$ & 2 & 3 & 4 & 5 \\
\hline \multicolumn{7}{|l|}{ Projeção Skyline } \\
\hline Radiopacidade de medular (grau) & - & $6,3 \%$ & $29,7 \%$ & $23,4 \%$ & $32,8 \%$ & $7,8 \%$ \\
\hline Irregularidades na cortical (grau) & $81,2 \%$ & $12,5 \%$ & $6,3 \%$ & $0 \%$ & - & - \\
\hline \multicolumn{7}{|l|}{ Projeção lateromedial } \\
\hline Irregularidades no contorno (grau) & $12,9 \%$ & $87,1 \%$ & $0 \%$ & $0 \%$ & - & - \\
\hline Relação corticomedular (grau) & $0 \%$ & $0 \%$ & $0 \%$ & $25,8 \%$ & $67,7 \%$ & $6,5 \%$ \\
\hline Entesiófitos no ligamento impar & $95,2 \%$ & $4,8 \%$ & - & - & - & - \\
\hline \multicolumn{7}{|l|}{ Projeção DPPD } \\
\hline Simetria (grau) & $17,7 \%$ & $59,7 \%$ & $22,6 \%$ & $0 \%$ & - & - \\
\hline Invaginações sinoviais (número) & $0 \%$ & $55,7 \%$ & $19,7 \%$ & $18,0 \%$ & $6,6 \%$ & $0 \%$ \\
\hline \multirow{2}{*}{ Borda proximal (forma) } & Reto & \multicolumn{2}{|c|}{ Côncavo } & Ondula & \multicolumn{2}{|c|}{ Convexo } \\
\hline & $33,3 \%$ & \multicolumn{2}{|c|}{$3,3 \%$} & $36,7 \%$ & \multicolumn{2}{|c|}{$26,7 \%$} \\
\hline
\end{tabular}

Na comparação entre grupos, não foi observado valor significativo no ponto de vista estatístico quanto à presença de irregularidades no contorno ósseo e à presença de entensiófitos no ligamento ímpar na exposição LM ou simetria óssea na exposição DPPD. Entretanto, diferenças estatísticas foram detectadas quanto à forma da borda proximal e quanto aos aspectos radiográficos em exposição SK. 
Conforme verificado na Fig. 1, ossos naviculares com a borda proximal côncava ou reta apresentaram maior espessura de medular em relação à cortical se comparados aos ossos naviculares com borda proximal convexa ou irregular. Também se verificou que ossos naviculares com irregularidades na borda flexora apresentaram maior escore para irregularidades de contorno ósseo em exposição LM (Fig. 2).

Quanto à radiopacidade da região medular em exposição SK, foi observado que ossos naviculares com radiopacidade aumentada (grau 5) apresentaram maior razão medular/cortical, maiores escores para relação corticomedular e maior número de invaginações sinoviais se comparados aos animais que apresentaram a região medular com rapiopacidade normal (graus 2-4; Fig. 3).

\section{DISCUSSÃO}

Dentre as características radiográficas estudadas, a radiopacidade da medular em relação à cortical na projeção Skyline foi a que mais se destacou. $\mathrm{Na}$ análise descritiva, apresentou $14,1 \%$ de anormalidades $(6,3 \%$ de radiopacidade reduzida e 7,8\% de radiopacidade aumentada), e na comparação com as outras características foi constatado que naviculares com radiopacidade medular aumentada possuem, estatisticamente, maior número de invaginações sinovais, maior espessura de cortical em relação à medular em

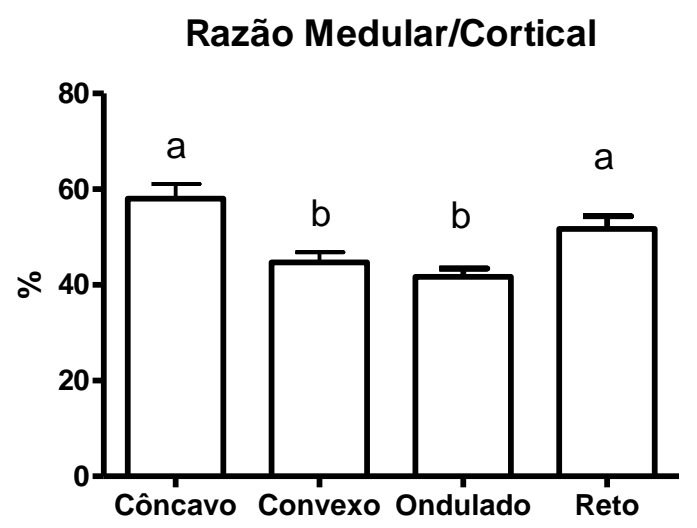

Figura 1. Médias e erros-padrão da razão medular/cortical de acordo com a forma da borda proximal dos ossos naviculares torácicos de equinos da Cavalaria Alferes Tiradentes. exposição Skyline e maior relação corticomedular em exposição LM.

$\mathrm{O}$ aumento de radiopacidade medular do osso navicular observada neste experimento tem sido associado a lesões crônicas, concordando com Pool (1995), as quais, como consequência, promovem um processo de remodelação e reabsorção óssea (Hernández e Novales, 1999; Clercq et al., 2000). Tais lesões, promovidas por trauma ósseo, também se manifestam pelo espessamento anormal das corticais e das lesões da cortical flexora, que dificilmente podem ser detectadas por radiologia convencional (Dyson, 2011), mas que explicam os valores superiores entre a razão medular/cortical e a relação corticomedular, observados em equinos com maior radiopacidade de medular (Fig. 3).

Segundo Stashak e Parks (2011), o exercício físico em superfícies duras aumenta a força por unidade de área no osso do navicular e no aparelho podotroclear, predispondo o aparecimento de lesões na região do osso do navicular. Tais observações estão de acordo com o trabalho exercido pela população aqui estudada, ou seja, equinos de cavalaria, a qual possui uso restrito em patrulhamento urbano, em vias pavimentadas. Do mesmo modo, Thompson et al. (1991) também observaram maior incidência de lesões do osso navicular em equinos que trabalham em piso duro.

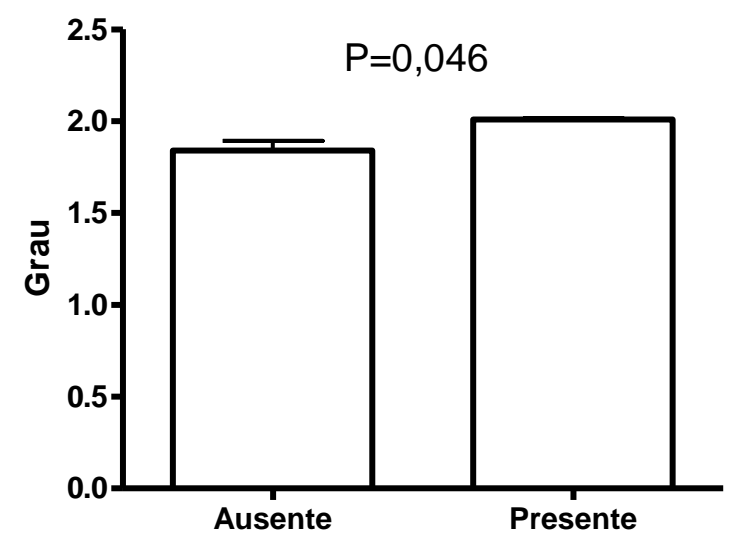

Figura 2. Médias e erros-padrão dos graus de irregularidades de contorno ósseo em naviculares torácicos de equinos da Cavalaria Alferes Tiradentes com presença ou não de irregularidades na cortical flexora. 
Razão Medular/Cortical

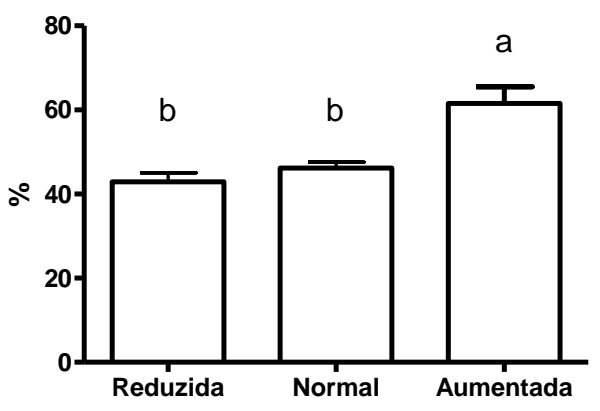

Relação Corticomedular

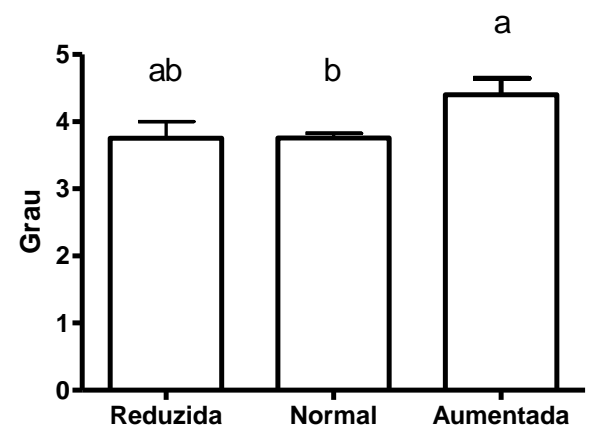

Invaginações sinoviais

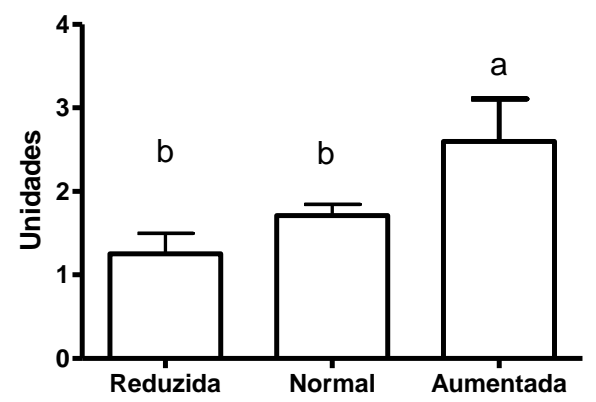

Figura 3. Médias e erros-padrão da razão medular/cortical dos graus de relação corticomedular e do número de invaginações sinoviais de acordo com a radiopacidade de medular em naviculares torácicos de equinos da Cavalaria Alferes Tiradentes.

No grupo em que foi observada a radiopacidade da medular aumentada, também foi observado um maior número de invaginações sinoviais (Fig. 3). Acredita-se que tais invaginações estejam relacionadas com o suprimento sanguíneo reduzido (Rijkenhuizen, 1989), e esse menor aporte sanguíneo pode ser resultante da compressão promovida pela bursa podotroclear e pelo tendão flexor digital profundo sobre essa região (Poulos, 1983). Postula-se que essas alterações são proporcionais à força e à vibração exercida pelo tendão flexor profundo sobre a superfície palmar do osso navicular, resultante do impacto do casco com o piso (Thompson et al., 1991). Animais de grande porte com cascos pequenos e o trabalho em piso duro são fatores correlacionados com maior número de invaginações sinoviais no osso navicular (Poulos, 1983; Thompson et al., 1991).

Quando observada a alteração do contorno do osso navicular, foi constatado que $12,9 \%$ dos animais apresentaram-se com grau de irregularidade 0 e foi observado grau de irregularidade 1 em $87,1 \%$ dos animais estudados neste experimento. Dik et al. (2001) e Rijkenhuizen (2006) descreveram que o formato do bordo proximal é geneticamente determinado e pode contribuir como fator predisponente para o desenvolvimento da síndrome do osso navicular, caracterizando-se como uma somatória para o aparecimento do quadro de síndrome do osso navicular quando os animais exercem atividade física em ambientes urbanos, onde predomina piso duro.

Quando avaliada a presença ou ausência de entesófitos no ligamento ímpar, não foi observado resultado significativo, Pottenger e Phillips (1990), Stashak (1994) e Doige e Hoffer (1983) não associaram essa alteração em equinos com histórico de síndrome do osso navicular, e sim como uma alteração característica de equino senil; por outro lado, Dyson e Kidd (1993) e Turner e Anderson (1993) relataram que o aparecimento de osteófito pode estar associado à enfermidade articular degenerativa da articulação interfalangeana distal.

\section{CONCLUSÃO}

A radiopacidade medular aumentada foi a principal alteração radiográfica detectada no aparato podotroclear de equinos de trabalho do Regimento de Cavalaria Alferes Tiradentes. Essa alteração está associada a maior número de invaginações sinoviais, a maior espessura de cortical em relação à medular em exposição Skyline e a maior relação corticomedular em exposição LM. Esses achados indicam uma predisposição desses equinos para desenvolver a síndrome do osso navicular. 


\section{AGRADECIMENTOS}

À Capes, à Fapemig e ao CNPq, pelo apoio financeiro; ao Regimento de Cavalaria Alferes Tiradentes da Polícia Militar do Estado de Minas Gerais, por permitir este estudo.

\section{REFERÊNCIAS}

BAKER, J.R.; ELLIS, C.E. A survey of post mortem findings in 480 horses 1958 to 1980: (1) causes of death. Equine Vet. J., v.13, p.43-46, 1981.

CLERCQ, T., VERSCHOOTEN, F.; YSEBAERT, M.A. 2000. Comparision of the palmaroproximalpalmarodistal view of the isolated navicular bone to otheir views. Vet. Radiol. Ultrasound, v. 41, p.525$533,2000$.

DABAREINER, R.M.; COHEN, N.D.; CARTER, G.K. et al. Lameness and poor performance in horses used for team roping: 118 cases (2000-2003). J. Am. Vet. Med. Assoc., v.226, p.1694-1699, 2005.

DOIGE, C.E.; HOFFER, M. A. Pathological changes in the navicular bone and associated structures of the horse. Can. J. Comp. Med., v.47, p.387-395, 1983.

DIK, K.; VAN DEN BELT, A.; VAN DEN BROEK, J. R. Relationships of age an shape of the navicular bone to the development of navicular disease: A radiological study. Equine Vet. J., v.33, p.172-175. 2001.

DYSON, S.J.; KIDD, L. A comparasion of responses to analgesia of navicular bursa and intra-articular analgesia of distal interphalangeal joint in 59 horses. Equine Vet. J., v. 25, p.93-98, 1993.

DYSON, S. Radiological interpretation of the navicular bone. Equine Vet. Educ., v.23, p.73- 82. 2011.

EGENVALL, A.; PENELL, J.C.; BONNETT, B.N. et al. Mortality of swedish horses with complete life insurance between 1997 and 2000: variations with sex, age, breed and diagnosis. Vet. Rec., v.158, p.397-406, 2006.

HERNÁNDEZ, E.M.; NOVALES, M. Diagnóstico radiológico del syndrome podotroclear. Imag. Vet., v. 2, p.66-68, 1999.

NUTRIENT requirements of horses. 6.ed. rev. Washington, D.C, 2007. 341 p.
PLEASANT, S.R.; CRISMAN, M.V. Navicular disease in horses: Pathogenesis and diagnosis. Vet. Med. Comp. Anim. Pract., v.92, p.250-257, 1997.

POOL, R.R. Pathophysiology of navicular disease. In: Proc. American Equine Sport Medicine, Davis, p. 444, 1995.

POTTENGER, L.A.; PHILLIPS, F.M. The effect of marginal osteophytes on reduction of varus-valgus instability in osteoarthritic knees. Arthritis Rheumatol., v.33, p.853-858, 1990.

POULOS, P.W. Correlation of the radiographic signs and histologic changes in navicular disease. In: ANNUAL CONVENTION OF THE AMERICAN ASSOCIATION EQUINE. PRACTITIONERS, 29., Las Vegas, 1983. Proceedings... Las Vegas: American Association Equine. Practitioners, 1983. p.241-255.

RIJKENHUIZEN, M.B.A. Navicular disease: a review of what's new. Equine Vet. J., v.38, p. 82-88, 2006.

RIJKENHUIZEN, M.B.A.; NEMETH, F.; DIK, J.K.; GOEDEGEBUURE, A.S. The arterial supply of the navicular bone in adult horses with navicular disease. Equine Vet. J., v.21. p.418-424, 1989.

RIVELLES, S. Policiais militares cumprindo suas verdadeiras funções. 2011.21f. Projeto temático (Especialização em Gestão Pública) - Universidade Federal do Paraná, Rio Negro, PR.

STASHAK, T. Doença do navicular (podotrocleose). In: STASHAK, T.S. Claudicação em eqüinos segundo Adams. São Paulo: Roca, 1994. Cap.8, p. 517-532.

STASHAK, T.S. PARKS, B.A. Lameness in the extremities. In: BAXTER, G.M. (Ed). Manual of equine lameness Hoboken, New Jersey: WilleyBlackwell, 2011. p.672-801.

THOMPSON, K.N.; ROONEY, J.R.; PETRITESMURPHY, M.B. Considerations on the pathogens of navicular disease. J. Equine Vet. Sci., v.11, p. 4-8. 1991.

TURNER, A.T.; ANDERSON, B. Lameness of the distal interphalangeal joint. Proc. 39th ANNUAL CONVENTION OF THE AMERICAN ASSOCIATION OF EQUINE PRACTITIONERS, 39., San Antonio, Texas, 1993. Proceedings... San Antonio: American Association of Equine Practitioners, 1983. p.252-257.

TURNER, T.A. 1990. Navicular disease: current practice of equine surgery. Philadelphia: W.B. Saunders, 1990. p.413-416. 